\section{Cardiac glycosides: a leap to lupus?}

\section{By Tracey Baas, Senior Editor}

A team from Harvard University and Columbia University College of Physicians and Surgeons has found that cardiac glycosides, a diverse family of sodium pump blockers that are used to treat heart failure and atrial arrhythmia, ${ }^{1}$ are also able to inhibit production of interferon- $\beta .^{2}$ The findings suggest that glycosides could be repurposed to treat inflammatory and autoimmune diseases, including lupus, that are characterized by aberrant production of interferons.

In an effort to develop compounds to treat inflammatory and autoimmune diseases caused by interferon (IFN) overproduction, the researchers set out to gain mechanistic insights into signaling pathways leading to interferon production.

They started by screening a small molecule library to identify compounds that inhibit virus-induced interferon- $\beta$ (IFNB; IFN- $\beta$ ) gene expression. The lead hit was bufalin. The group also confirmed that two other cardiac glycosides, ouabain and digoxin, inhibited IFN- $\beta$.

Lanoxin digoxin is marketed by GlaxoSmithKline plc as a cardiac antiarrhythmic to treat congestive heart failure and cardiac arrhythmia. It replaced ouabain therapy because of its superior bioavailability.

In cultured cells infected with virus or treated with double-stranded RNA or double-stranded DNA to induce IFN, bufalin blocked IFN- $\beta$ production and signaling compared with vehicle control.

The researchers then asked whether inhibition was due to the interaction of bufalin with the sodium pump itself or from some other off-target activity. The answer was the former: In cells expressing a drug-insensitive variant of the $\mathrm{Na}^{+} / \mathrm{K}^{+}$ATPase pump, bufalin had no effect on IFN- $\beta$ production. Also, short hairpin RNA knockdown of the pump inhibited IFN- $\beta$ production and signaling.

Further in vitro work teased out the precise mechanism by which blocking the pump dampens IFN levels. Cardiac glycoside-mediated increases in intracellular $\mathrm{Na}^{+}$inhibit the ATPase activity of DEAD box polypeptide 58 (DDX58; RIG-I), an early and essential component in the pathway of type I IFN production (see Figure 1, "Blocking interferon in lupus").

The team was led by Tom Maniatis, professor of biochemistry at Columbia University College of Physicians and Surgeons. Results were published in Nature Chemical Biology.

\section{Leap to lupus}

The team now plans to test the idea in mouse models. But testing cardiac glycosides in those animals is not straightforward because

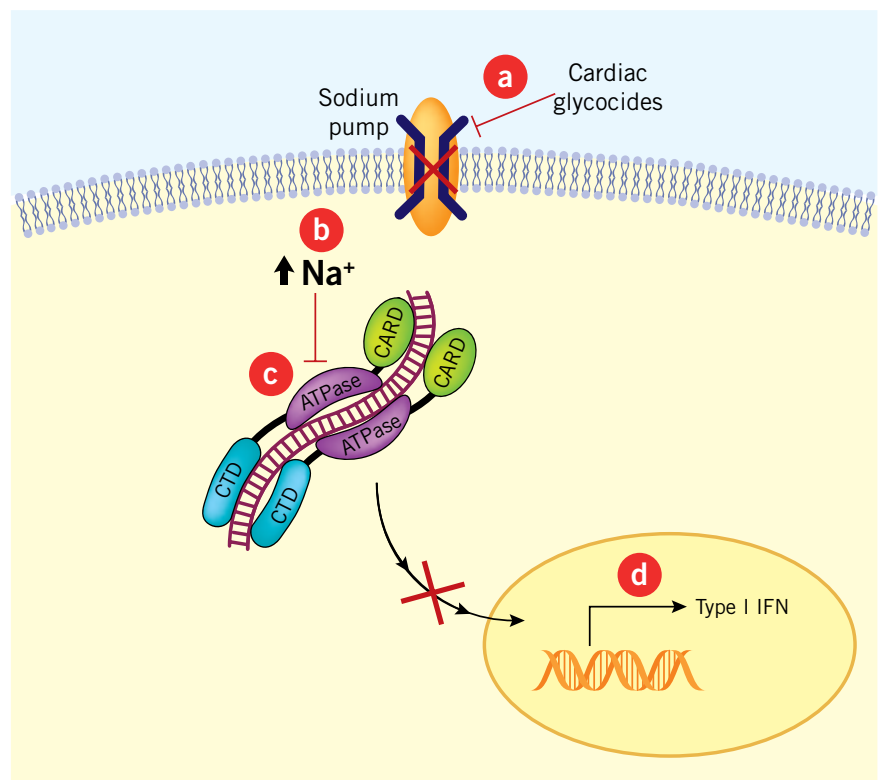

Figure 1. Blocking interferon in lupus. Cells infected with virus or treated with double-stranded DNA or double-stranded RNA produce type I interferon (IFN), an essential component of the innate immune response. Ye et al. show that treating these cells with cardiac glycosides [a] blocks the $\mathrm{Na}^{+} / \mathrm{K}^{+}$ATPase pump to increase intracellular levels of $\mathrm{Na}^{+}[\mathbf{b}]$. This results in inhibition of the ATPase activity of DEAD box polypeptide 58 (DDX58; RIG-I) [c], an essential regulator of type I IFN production [d].

RIG-I contains caspase activation and recruitment domains (CARDs), ATPase domains and carboxyl-terminal domains (CTDs).

Because overproduction of type I IFN is a critical factor in the autoimmune disease systemic lupus erythematosus (SLE), the researchers suggest that targeting the ATPase activity of RIG-I might be potentially useful to treat lupus.

Although viral infection is known to induce IFN production through RIG-I by way of double-stranded RNA and possibly double-stranded DNA (dsDNA), the nature of IFN inducers in lupus is not clear. Ribonucleoprotein complexes, dsDNA and chromatin have all been implicated as IFN inducers, most notably through toll-like receptors (see Figure 1 in Fulmer, T., SciBX 3(26); doi:10.1038/scibx.2010.784). RIG-I is involved in sensing these IFN inducers in various cell types, but whether it also does so in lupus is not known.

wild-type mice harbor a sodium pump that is intrinsically insensitive to cardiac glycosides.

To get around that, Maniatis told SciBX, "we have obtained a mutant mouse from Dr. Jerry Lingrel, professor in the Department of Molecular Genetics, Biochemistry and Microbiology at the University of Cincinnati, in which the appropriate mutation has been knocked in to make the mice sensitive" to the compounds. ${ }^{3}$ "By crossing various disease models into that background it would 
be possible to do in vivo studies. We are most interested in mouse models of lupus."

One issue that needs to be resolved, according to Keith Elkon, is that although cardiac glycosides "have the potential to modulate IFN- $\beta$ expression in vivo," it remains unclear whether their effects on RIG-I will dampen type I IFN production in a disease setting. Elkon is professor of medicine and head of rheumatology at the University of Washington.

"RIG-I has not been clearly implicated in expression of type I IFN in diseases such as [systemic lupus erythematosus]," he added. "The only pathway that we currently understand to generate the IFN signature in lupus is the stimulation of toll-like receptors."

Even if blocking RIG-I does lead to decreased expression of IFN- $\beta$ in lupus, Elkon said it may not confer immediate benefit. "While it is well known that IFN- $\beta$ primes for the production of IFN- $\alpha$, by the time we see the patient they are already making IFN- $\alpha$, so blockade of IFN- $\beta$ may not be beneficial in the short term. That is why current clinical trials target IFN- $\alpha$," he said.

Thus, Daniel Zagury, professor at Pierre and Marie Curie University and founder of Neovacs S.A., wanted to see how cardiac glycosides affect interferon- $\alpha$ (IFNA; IFN- $\alpha$ ) production and signaling. "If cardiac glycosides also block IFN- $\alpha$ signaling effects, they could represent a therapeutic candidate for systemic lupus erythematosus," he told SciBX.

Neovacs' IFN $\alpha$-Kinoid, a cytokine derivative conjugated to a carrier protein, is in Phase I/II testing to treat lupus. Anti-IFN- $\alpha$ antibodies in Phase II testing for lupus include MED-545 from AstraZeneca plc and Bristol-Myers Squibb Co. and RG7415 from Roche's Genentech Inc. subsidiary.

Maniatis thinks cardiac glycosides are likely to block IFN- $\alpha$ in vivo. "In most human cells other than lymphocytes, IFN- $\beta$ is the only IFN gene expressed in response to infections, which is the focus of our paper. However, we do provide one experiment that shows cardiac glycosides inhibit the expression of both IFN- $\alpha$ and IFN- $\beta$ in a human B cell line. Of course, both are induced by a common pathway that requires RIG-I."

Because IFN- $\beta$ treatment in multiple sclerosis patients has been shown to lead to lupus-like symptoms in some instances, ${ }^{4}$ Maniatis noted, "there is precedent for further investigation of type I interferon induction and blockade, both IFN- $\alpha$ and IFN- $\beta$, in lupus mouse models."

\section{Heart of the matter}

Even though glycosides are used to treat cardiovascular disease, they can cause side effects including irregular heartbeat and dosedependent toxicity.
Zagury would like to see preclinical experiments to find out if these occur, especially within the context of glomerulonephritis. Lupus patients often develop the condition, which has been shown to contribute to cardiac complications.

Maniatis agreed: "Because cardiac glycosides have been used for so many years, side effects are well known and can be serious. Clinical trials will clearly be required to find optimal dosing."

Elkon pointed out that "cardiac glycosides such as digoxin have been used to treat occasional cardiac disease in SLE without any adverse effect to my knowledge. However, one would need to exercise caution in view of reports of glycosides causing increased apoptosis and raised blood pressure in experimental models."

Indeed, any potential dosing issues with bufalin itself are largely unknown because the compound has not been used in a cardiovascular setting.

"The primary concern of applying bufalin as a therapeutic agent is whether patients can tolerate the amount of drug necessary to inhibit the production of IFN," Maniatis said. "A published study of bufalin treatment for cancer patients ${ }^{5}$ showed that the effective concentration of bufalin we used in our study to inhibit IFN was tolerated in the cancer patients. This suggests that bufalin could in principle be used to treat SLE patients."

A patent application has been filed for inflammatory and autoimmune diseases and is available for licensing from Harvard University.

Baas, T. SciBX 3(46); doi:10.1038/scibx.2010.1372

Published online Dec. 2, 2010

\section{REFERENCES}

1. Prassas, I. \& Diamandis, E.P. Nat. Rev. Drug Disc. 7, 926-935 (2008)

2. Ye, J. et al. Nat. Chem. Biol.; published online Nov. 14, 2010; doi:10.1038/nchembio.476

Contact: Tom Maniatis, Columbia University College of Physicians and Surgeons, New York, N.Y.

e-mail: tm2472@mail.cumc.columbia.edu

3. Wansapura, A.N. et al. Am. J. Physiol. Heart Circ. Physiol.; published online Oct. 15, 2010; doi:10.1152/ajpheart.00625.2010

4. Javed, A. \& Reder, A.T. Pharmacol. Ther. 110, 35-56 (2006)

5. Meng, Z. et al. Cancer 115, 5309-5318 (2009)

COMPANIES AND INSTITUTIONS MENTIONED

AstraZeneca plc (LSE:AZN; NYSE:AZN), London, U.K. Bristol-Myers Squibb Co. (NYSE:BMY), New York, N.Y. Columbia University College of Physicians and Surgeons. New York, N.Y.

Genentech Inc., South San Francisco, Calif.

GlaxoSmithKline plc (LSE:GSK; NYSE:GSK), London, U.K. Harvard University, Cambridge, Mass.

Neovacs S.A. (Euronext:ALNEV), Paris, France

Pierre and Marie Curie University, Paris, France

Roche (SIX:ROG; OTCQX:RHHBY), Basel, Switzerland

University of Cincinnati, Cincinnati, Ohio

University of Washington, Seattle, Wash. 